\title{
Early Detection of Cheyne-Stokes Breathing via ECG-Derived Respiration in Patients with Severe Heart Failure: a Pilot Study
}

\author{
Pauline Guyot $^{1,2,3}$, Bruno Chenuel ${ }^{4}$, El-Hadi Djermoune ${ }^{1}$, Thierry Bastogne B $^{1,2,3}$ \\ ${ }^{1}$ CRAN CNRS UMR 7039, BP 70239, F-54506 Vandoeuvre-lès-Nancy Cedex, France, \\ ${ }^{2}$ INRIA BIGS, BP 70239, 54506 Vandoeuvre-lès-Nancy Cedex, France, \\ ${ }^{3}$ CYBERnano, 193 av. Paul Muller, 54602 Villers-lès-Nancy \\ ${ }^{4}$ EA 3450 DevAH, 54602 Villers-lès-Nancy
}

\begin{abstract}
We present in this paper a preliminary study for detecting early pattern of Cheyne-Stokes Breathing using a single electrocardiogram signal in patients with severe heart failure. Two ECG-derived respiration signals, namely Heart-Rate and R-Wave Amplitude, are computed and jointly used to estimate different respiratory events, respiratory rate and amplitude modulation. Three patients whose respiration goes from normal to severe CSB are used to test our method. Results show good performance for the detection of breathing cycles compared with the ventilation signal and the final classification based on respiratory events, AHI, amplitude modulation reveals exact correlation with the expert.
\end{abstract}

\section{Introduction}

Cheyne-Stokes Breathing (CSB) is a type of sleepdisordered respiration characterized by a crescendodecrescendo pattern of ventilation, alternating hyperventilation and central hypopneas/apneas. CSB is mainly prevalent in patients with severe heart failure (ventricular ejection fraction less than 30\%) and can be associated with a worse prognosis [1]. Overnight multichannel polysomnography is commonly used for diagnosis [2] and for the identification of patients with periodic breathing preceding CSB. Unfortunately, this is a laborintensive and expensive method.

To get around polysomnography, several portable systems have been developed in the last 20 years like single lead photoplethysmograph (PPG) [3], oximetry [4] or simple respiratory recording [5]. Continuous electrocardiogram (ECG) signals have also been used to extract respiratory signal [6] and to detect sleep apneas [7] and hypopneas [8]. The construction of such ECG-Derived Respiration (EDR) relies on the change of distance between ECG electrodes and the heart during respiration. It creates a modulation in the QRS morphology and a respiration signal can be extracted using R-Wave Amplitude (RWA). Also, respiration has an impact on Heart Rate (HR) as the sinus node is excited by the autonomic nervous system which increases during inhalation and decreases during exhalation [9]. Therefore, a respiratory signal can be extracted from the HR signal.

Our objective is to develop a new method to detect early stages of CSB based on a single electrocardiogram signal and to demonstrate the proof of concept on three patients. The proposed approach uses temporal [10] and frequential features extracted from two EDRs (HR and RWA), a classification of epochs and a pre-diagnostic decision are then computed.

\section{Methodology}

\subsection{Patients}

The study involves three patients, all presenting heart failure with low ejection fraction $(\mathrm{EF}<30 \%)$. They present different type of breathing: patient $\mathrm{n}^{\circ} 1$ (age 59 y.o. with a Body Mass Index of $30.2 \mathrm{~kg} / \mathrm{m}^{2}$ and an EF of $25 \%$ ) has a normal respiration and exhibits no respiratory pathology, patient $\mathrm{n}^{\circ} 2$ (age 61 y.o. with a BMI of $33.4 \mathrm{~kg} / \mathrm{m}^{2}$ and an EF of $25 \%$ ) presents a periodic breathing likely preceding CSB and patient $\mathrm{n}^{\circ} 3$ (age 80 y.o. with a BMI of $35.8 \mathrm{~kg} / \mathrm{m}^{2}$ and an EF of 28\%) shows a severe Cheyne-Stokes respiration (CSB-CSA) with an apnea-hypopnea index (AHI) of $56 / \mathrm{h}$. Diagnostic is established by a pulmonologist and written consent was obtained for all participants.

\subsection{Experiments}

Ventilation signal $\left(\dot{V}_{E}\right)$ was measured along with endtidal carbon dioxide $\left(\mathrm{ETCO}_{2}\right)$ and oxygen $\left(\mathrm{ETO}_{2}\right)$. Ambulatory electrocardiogram (ECG), oxygen saturation, thoracic belt respiration and blood pressure were also 
simultaneously recorded. Subjects were placed in supine position on a bed for about 30 minutes of recording.

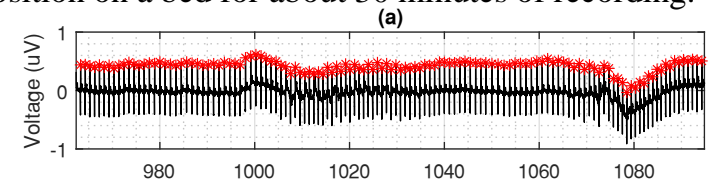

(b)



(c)

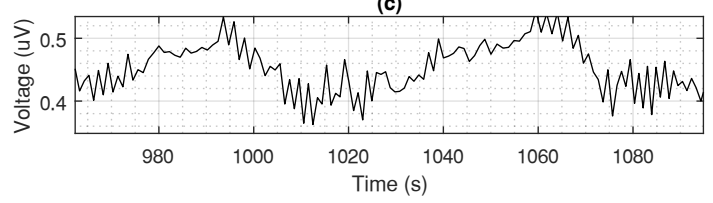

Figure 1. ECG-Derived Respiration construction for the patient $\mathrm{n}^{\circ} 3$ exhibiting a severe Cheyne-Stokes breathing (a) original ECG (lead V3) with annotated peaks (b) Heart-Rate signal (c) R-Wave Amplitude signal.

\subsection{Diagnostic criteria for CSR}

Cheyne-Stokes respiration was defined by the presence of the classical pattern of waxing/waning in the tidal volume with central hypopneas/apneas. Central hypopnea was defined as a reduction of tidal volume of $30 \%$ along with a drop of $3 \%$ in oxygen saturation - if no flow limitation or obstructive apnea is observed. Central apnea was defined as a cessation of tidal volume for at least 10 seconds without any respiratory efforts.

\subsection{EDR extraction}

ECG signal was firstly filtered to remove power line interference $(50 \mathrm{~Hz})$. R peaks are automatically detected using Non-Negative Matrix Factorization [11]. Two ECGDerived Respiration (EDR) signals are computed to extract respiratory signal from ECG. Heart-Rate (HR) is computed directly from the detection, whereas R-Wave Amplitude (RWA) requires the suppression of the baseline wander [12] through median filtering (200 ms). Both signals are evenly resampled with linear interpolation (Figure 1).

\subsection{Estimation of breathing cycles}

Change point analysis (CPA) is used to detect breathby-breath respiration from EDR. If we consider the signal $x \in \mathbb{R}^{N}$, we assume that some statistical properties change abruptly at instants $t_{1}, \ldots, t_{K}$, called change points. In CPA methods, the aim is to estimate the segmentation $\hat{t}=\left\{\widehat{t_{1}}, \widehat{t_{2}}, \ldots\right\}$ through the minimization of a cost function $C$ which represents the total residual error of the statistical estimate for each section. When the number of changes $K$ is unknown, a penalty term is added to the residual error: $\min _{t} C(t)+\beta K$ with $\beta>0$. In our case, a change point represents a peak or a trough of signal (inspiration and expiration) and the statistical properties used are slope and mean. Once all change points are detected, slope is computed for all sections and those lower than a threshold experimentally set to $10^{-3}$ are discarded and considered as noise. Finally, only peaks whose section duration is greater than 1 second are conserved (biologic a priori: respiratory rate between 12 and 20 cycles per minute) (Figure 2).

\subsection{Classification of respiratory events}

A first temporal analysis of EDR signals is realized to extract respiratory metrics (Table 1). Apneas are detected as a cessation of respiratory peaks of more than 10 seconds. If apneas are detected, signal is fragmented in cycles (from the end of an apnea to the end of the next one) and several features are computed: cycle length $(\mathrm{CL})$, apnea length (AL) and ventilation length (VL) (Figure 2). A first score $S_{t}$ is attributed to each segment according to its features, corresponding to its characterization for CSB (Table 1).

(a)



(b)

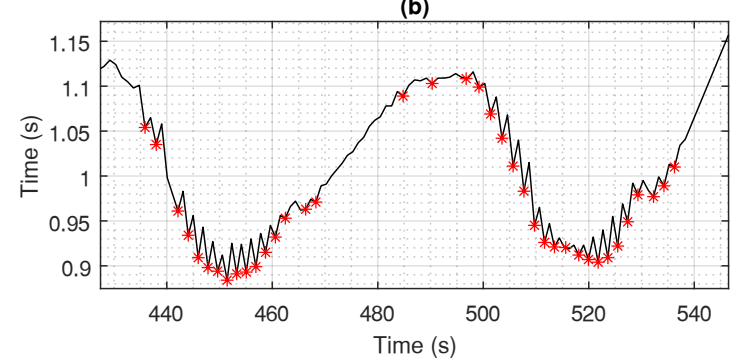

Figure 2. ECG-Derived Respiration for the patient $n^{\circ} 3$ (severe CSB) (a) Ventilation signal with durations: VL corresponding to Ventilation Length, AL to Apnea Length and CL to Cycle Length (b) Heart-Rate signal with annotated expiration.

\begin{tabular}{|c|c|}
\hline Score $S_{t}$ & Particularity \\
\hline 3 & Very likely CSB pattern \\
\hline 2 & Potential CSB pattern \\
\hline 1 & Very unlikely CSB pattern \\
\hline
\end{tabular}

Table 1. Temporal score $S_{t}$, computed as the number of following assessments true (*) $30<C L<120$, (**) $10<A L<40$ and (***) $20<V L<80$.

Then, a frequential analysis of both EDR signals is performed. Signals are divided into epochs of 2 minutes 
and Fast Fourier Transform is applied to find its main peak frequency $f_{p}$. A second score $S_{f}$ is set to 3 if $f_{p}$ is in the typical pathological interval of CSB for both signals (between $0.008 \mathrm{~Hz}$ and $0.03 \mathrm{~Hz}$, for a cycle length from 30 seconds to 2 minutes) and 2 for only one signal out of the both. For each segment, FFT of the derivative of the signal is also computed to distinguish CSB and obstructive pattern (crescendo/decrescendo of CSB is assimilated to a sine wave and obstructive event to a square signal). Main peak frequency and its potential harmonics are detected. If the amplitude ratio between the main peak and its first detected harmonic is more than $1 / 3$, the segment is considered to be an obstructive event and its score is set to 1. Finally, if no pattern is detected, the score is set to 0 (Table 2).

\begin{tabular}{|c|c|}
\hline Score $S_{f}$ & Particularity \\
\hline 3 & CSB pattern \\
\hline 2 & Potential CSB pattern \\
\hline 1 & Obstructive pattern \\
\hline 0 & Normal breathing \\
\hline
\end{tabular}

Table 2. Frequential score $S_{f}$, computed as follows: (3) HR and RWA main frequencies in the typical pathological interval of CSB, (2) one signal out of both in the typical pathological interval of CSB, (1) first harmonic amplitude significantly high to be an obstructive event and (0) no pattern detected.

The frequential score concerns potential amplitude modulation in the ventilation whereas the temporal score informs on the presence of apneas and on the temporal characteristics of the cycles [10]. Jointly used, the scores can provide a classification (Table 3) for each segment between (1) normal breathing, (2) obstructive hypopneas, (3) obstructive apneas, (4) periodic breathing (CSB hypopneas) (5) potential CSB apneas and (6) CSB apneas. A final decision leads to a pre-diagnostic using the number of events of each category.

\begin{tabular}{|c|c|c|c|c|c|}
\cline { 3 - 6 } \multicolumn{2}{c|}{} & \multicolumn{4}{|c|}{ Frequential score } \\
\cline { 3 - 6 } \multicolumn{2}{c|}{} & 0 & 1 & 2 & 3 \\
\hline \multirow{3}{*}{ 范 } & 0 & $(1)$ & $(2)$ & $(4)$ & $(4)$ \\
\cline { 2 - 6 } & 1 & - & $(3)$ & $(5)$ & $(6)$ \\
\cline { 2 - 6 } & 2 & - & $(3)$ & $(5)$ & $(6)$ \\
\cline { 2 - 6 } & 3 & - & $(3)$ & $(5)$ & $(6)$ \\
\hline
\end{tabular}

Table 3. Classification of epochs into respiratory events.

\section{Results}

Detection of breathing cycles in both EDR signals is compared to the respiration signal in Table 4. Our method achieves better results for RWA than HR in all cases except for the patient with severe CSB-CSA. For this patient, RWA detects more True Positives than HR but tends to over-detect respiration when the patient is in apnea. This phenomenon is highlighted in Table 5 where the results of the detection of apneas in EDR signals are listed. Clearly, HR over-performs RWA, as most of the False Positives of RWA are detected during apneas. Therefore, HR is privileged in the characterization of the temporal analysis.

\begin{tabular}{|c|c|c|c|c|c|c|}
\cline { 2 - 8 } \multicolumn{1}{c|}{} & \multicolumn{2}{c|}{$\begin{array}{c}\text { Normal } \\
\text { breathing } \\
\text { Patient }{ }^{\circ} 1\end{array}$} & \multicolumn{2}{c|}{$\begin{array}{c}\text { CSR } \\
\text { Patient } \mathrm{n}^{\circ} 2\end{array}$} & \multicolumn{2}{c|}{$\begin{array}{c}\text { CSB-CSA } \\
\text { Patient } \mathrm{n}^{\circ} 3\end{array}$} \\
\cline { 2 - 8 } \multicolumn{1}{c|}{} & $H R$ & $R W A$ & $H R$ & $R W A$ & $H R$ & $R W A$ \\
\hline $\mathrm{TP}$ & 448 & 467 & 334 & 349 & 464 & 486 \\
\hline $\mathrm{FP}$ & 25 & 42 & 75 & 47 & 80 & 122 \\
\hline $\mathrm{FN}$ & 20 & 1 & 27 & 12 & 38 & 16 \\
\hline Se & 95.72 & 99.78 & 92.52 & 96.67 & 92.43 & 96.81 \\
\hline Pr & 94.71 & 91.74 & 81.66 & 88.13 & 85.29 & 79.93 \\
\hline Acc & 90.78 & 91.56 & 76.60 & 85.53 & 79.72 & 77.78 \\
\hline $\begin{array}{l}\text { Total } \\
\text { Beats }\end{array}$ & \multicolumn{3}{|c|}{468} & \multicolumn{2}{|c}{361} & \multicolumn{2}{c|}{502} \\
\hline
\end{tabular}

Table 4. Detection of breathing cycles in Heart-Rate and R-Wave Amplitude signals compared to ventilation signal. TP, FP and FN mean True Positives, False Positives and False Negatives respectively. Se, Pr and Acc correspond to Sensitivity, Precision and Accuracy respectively.

\begin{tabular}{|c|c|c|c|}
\cline { 2 - 4 } \multicolumn{1}{c|}{} & TP & FP & FN \\
\hline HR & 25 & 0 & 2 \\
\hline RA & 7 & 0 & 20 \\
\hline
\end{tabular}

Table 5. Detection of apneas in ECG-Derived Respiration for patient $\mathrm{n}^{\circ} 3$ (severe CSB).

The temporal score $S_{t}$ is computed with features extracted from cycles between two apneas. For patient $\mathrm{n}^{\circ} 3$, cycle length (CL) is estimated to $71.06 \pm 44.02$ seconds in HR signal $(64.90 \pm 5.04$ seconds for respiratory signal), apnea length $(\mathrm{AL})$ to $14.79 \pm 2.42$ seconds $(29.24 \pm 4.09$ seconds for respiratory signal) and ventilation length (VL) to $56.26 \pm 43.42$ seconds $(35.66 \pm 3.98$ seconds for respiratory signal). CL is well estimated, but AL is underestimated while VL is over-estimated. This is due to a misdetection of breathing in presence of apnea, shortening its estimated duration. It leads to a correct scoring for the temporal analysis in 16 cases out of 26 (16 cycles are scored as 3,2 cycles as 2 and 6 cycles as 1 , when for the respiration signal, all cycles are scored as 3 ).

To analyze the frequential score $S_{f}$, the same analysis was run on the respiration signal with 3 levels $(0$ is for normal breathing, 1 is for obstructive pattern and 2 for CSB pattern). The frequential analysis of the HR signal shows high correlation with the respiration signal. For the patient $\mathrm{n}^{\circ} 1$, all epochs are scored as 0 for both configurations (EDRs and ventilation). For the patient $\mathrm{n}^{\circ} 2,10$ epochs are scored as 2 (potential CSB pattern) for the EDRs while 12 epochs are classified as CSB pattern. For the patient $n^{\circ} 3$, all epochs are scored as 3 (CSB pattern) for the EDRs and 2 for respiratory signal (CSB pattern). 
Finally, pre-diagnostic shows exact correlation with the expert: patient $\mathrm{n}^{\circ} 1$ is classified as normal breathing with no cycle detected and all epoch scored as 0 , patient $n^{\circ} 2$ is classified as CSB pattern with no apnea detected and 10 out of 16 epochs with potential CSR pattern and patient $\mathrm{n}^{\circ} 3$ is classified as CSB-CSA with 26 cycles detected with very likely CSB pattern and all epochs scored as CSB pattern.

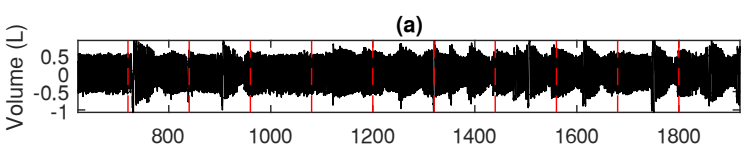

(b)


Figure 2. Results of scoring and classification for patient $\mathrm{n}^{\circ} 2$ (a) Ventilation with epochs of 2 minutes (b) Frequential score $S_{f}$ (c) Respiratory events detected by our method (see Table 3), 9 epochs are classified as CSB hypopneas and 2 epochs as normal breathing, (d) Respiratory events detected by the expert on the ventilation signal, 9 epochs are classified as CSB hypopneas and 2 epochs as normal breathing. 2 epochs are misclassified by our method.

\section{Conclusion}

A new computational method was developed to detect Cheyne-Stokes Breathing patterns via ECG-derived respiration in patients with severe heart failures. The proof of concept was demonstrated on three patients, all presenting heart failure with low ejection fraction but with different CSB status (no CSB, CSB and CSB-CSA). Results are promising and show the ability of the diagnostic method to detect early stages of CSB using a single electrocardiogram. The proposed method has now to be validated on a wider range of patients, but such a method could be employed as a powerful screening tool for early diagnosis and treatment of CSB in severe heart failure.

\section{Acknowledgements}

The authors wish to thank the virtual FEDER hospital project of Lorraine, France (FEDER-Etat-Région Hôpital Virtuel de Lorraine) for their valuable support.

\section{References}

[1] T. Brack et al., Daytime Cheyne-Stokes respiration in ambulatory patients with severe congestive heart failure is associated with increased mortality, Chest, vol. 132, $\mathrm{n}^{\mathrm{O}} 5$, p. 1463-1471, nov. 2007.

[2] M. T. L. Rovere et al., Clinical relevance of short-term day-time breathing disorders in chronic heart failure patients, European Journal of Heart Failure, vol. 9, n 9 , p. 949-954.

[3] O. Amir, D. Barak-Shinar, A. Henry, and F. W. Smart, Photoplethysmography as a single source for analysis of sleep-disordered breathing in patients with severe cardiovascular disease: SDB analysis from PPG signal, Journal of Sleep Research, vol. 21, nº 1, p. 94-100, Feb. 2012.

[4] C. Y. Lau and J. P. Armitstead, Discrimination of cheynestokes breathing patterns by use of oximetry signals, US20120016218A1, 19-jan-2012.

[5] J. Armitstead, Method for detecting and discriminating breathing patterns from respiratory signals, US8066647B2, 29-nov-2011.

[6] P. H. Charlton, T. Bonnici, L. Tarassenko, D. A. Clifton, R. Beale, and P. J. Watkinson, An assessment of algorithms to estimate respiratory rate from the electrocardiogram and photoplethysmogram, Physiol. Meas., vol. 37, $\mathrm{n}^{\circ}$ 4, p. 610, 2016.

[7] T. Penzel, J. McNames, P. De Chazal, B. Raymond, A. Murray, and G. Moody, Systematic comparison of different algorithms for apnoea detection based on electrocardiogram recordings, Medical and Biological Engineering and Computing, vol. 40, $\mathrm{n}^{\mathrm{o}} 4$, p. 402-407, 2002.

[8] V. Pichot et al., ECG-derived respiration: A promising tool for sleep-disordered breathing diagnosis in chronic heart failure patients, International Journal of Cardiology, vol. 186, p. 7-9, May 2015.

[9] M. H. Imam, C. K. Karmakar, A. H. Khandoker, and M. Palaniswami, Effect of using ECG derived respiration (EDR) signal in linear parametric QT-RR modeling, in 2013 35th Annual International Conference of the IEEE Engineering in Medicine and Biology Society (EMBC), 2013, p. 1968-1971.

[10] J. Wedewardt, T. Bitter, C. Prinz, L. Faber, D. Horstkotte, and O. Oldenburg, Cheyne-Stokes respiration in heart failure: Cycle length is dependent on left ventricular ejection fraction, Sleep Medicine, vol. 11, n ${ }^{\circ}$ 2, p. 137-142, feb. 2010.

[11] P. Guyot, P. Voiriot, E.H. Djermoune, S. Papelier, C. Lessard, M. Felices and T. Bastogne, R-peak detection in Holter ECG signals using Non-Negative Matrix Factorization, Computing in Cardiology, 2018.

[12] P. de Chazal, M. O'Dwyer, and R. B. Reilly, Automatic classification of heartbeats using ECG morphology and heartbeat interval features, IEEE Transactions on Biomedical Engineering, vol. 51, no. 7, pp. 1196-1206, Jul. 2004.

Address for correspondence.

Pauline Guyot

CRAN - Campus Science

Boulevard des Aiguillettes

54506 Vandoeuvre-lès-Nancy

Mail : pauline.guyot@univ-lorraine.fr 